$53.8 \%$ were iatrogenic preterm deliveries. There was an incidence of pre-eclampsia of $11.9 \%$ and $10.7 \%$ of the pregnancies were complicated by fetal growth restriction. The percentage of caesarean delivery was $40.5 \%$. $10.7 \%$ of neonates had criteria of neonatal lupus, and there was one case of congenital complete heart block, which required a neonatal cardiac pacemaker. There were no cases of neonatal deaths or asphyxia.

Conclusions In SLE pregnant patients, to ensure the best maternal and fetal outcomes, it is crucial that the pregnancy occurs in a period of immunological stability as well as an adequate surveillance by a multidisciplinary team prepared to control all the complications that may arise.

\section{P72 KNOWLEDGE AND USE OF CONTRACEPTIVE METHODS IN PORTUGUESE WOMEN WITH SYSTEMIC LUPUS ERYTHEMATOSUS}

${ }^{1}$ Luisa Brites, 'Stefanie Silva, 2,3 Laura Andreoli, 1,4Luis Inês. 'Rheumatology Dept., CHUC, Coimbra, Portugal; ${ }^{2}$ Dept. of Clinical and Experimental Sciences, University of Brescia, Brescia; ${ }^{3}$ Unit of Rheumatology and Clinical Immunology, ASST Spedali Civili, Brescia, Italy; ${ }^{4}$ Faculdade Ciências da Saúde-UBI, Covilhã, Portugal

\subsection{6/lupus-2020-eurolupus.117}

Background Systemic lupus erythematosus (SLE) affects women of childbearing age. Therefore contraceptive/family counselling are crucial to avoid unintended pregnancies. Our aim was to identify unmet needs for contraceptive/family counselling in women with SLE.

Methods Cross-sectional study including women aged 18-45 year-old fulfilling ACR 1997/SLICC criteria. All patients signed an informed consent and fulfilled a questionnaire with 15 short answers questions evaluating 4 domains: brief obstetric history; knowledge about the relationship between pregnancy and SLE, contraceptive/family counselling, contraception use and type of medical care offered. A descriptive analysis was used to summarize demographic/clinical data; possible predictors of contraceptive use (age, previous spontaneous abortion, level of knowledge about SLE, contraceptive/ family planning) were tested by multiple regression analysis using SPSS Statistics, V.21; $p<0.05$ was considered statistically significant.

Results We enrolled 108 women (mean age 34.4 \pm 7.1 years; mean disease duration $10.3 \pm 7.3$ years). About $65 \%$ of the included patients received information about family planning (mostly from rheumatologists $(62.9 \%)$ ) and $81 \%$ received information about contraception (mostly from gynaecologists $(56.3 \%))$. Only $38 \%$ was considered informed about SLE and its influence in pregnancy. In this cohort, $23.1 \%$ wanted to get pregnant in the next 6 months; the rest of them already had the number of children they wanted or wanted to get pregnant later. Contraceptive use was reported by $79.6 \%$ of the patients and the most commonly used was oral contraceptive pills. Of those who had no contraception method, $60 \%$ admitted having unprotected sex. No statistically significant predictors of contraceptive use were identified.
Conclusion In this tertiary Lupus Clinic, most patients received effective contraceptive/family counselling and use contraceptive methods. Quality of the given information can still be improved.

\section{P73 THE RELATIONSHIP BETWEEN PREGNANCY, DISEASE ACTIVITY AND ADVERSE PREGNANCY OUTCOMES IN SYSTEMIC LUPUS ERYTHEMATOSUS}

Çiğdem Çetin', Tuğba Saraç-Sivrikoz², Müge Ateş-Tikiz², Sibel Zarali', Ayşenur Ersoy ${ }^{1}$ Yasemin Yalçinkaya', Ahmet Gül', Lale Öcall', Murat İnanç', İbrahim Kalelioğlu², Bahar Artim-Esen'. 'Istanbul Faculty of Medicine, Dept. of Internal Medicine, Division of Rheumatology, Istanbul; ${ }^{2}$ Istanbul Faculty of Medicine, Dept. of Obstetrics and Gynecology, Istanbul, Turkey

\subsection{6/lupus-2020-eurolupus.118}

Objective SLE can present with disease flares during pregnancy and postpartum period resulting in adverse pregnancy outcomes (APO). Herein we aimed to determine the effects of pregnancy on disease activity and the correlation of disease flares and APO.

Methods A total of 168 pregnancies involving 136 patients were included. Clinical and laboratory findings were described and disease activity was calculated using SLEDAI-2K (in the preconceptional period, all trimesters and postpartum). Flares and patients with low lupus disease activity scores(LLDAS) during each of these periods were identified. Fetal/neonatal death, premature birth due to preeclampsia, eclampsia or HELLP syndrome, neonates small for gestational age were described as APO and its relation to disease activity was studied

Results Mean SLEDAI-2K scores was $1.3 \pm 2.2(0-16)$ in the preconceptional period, $1.7 \pm 3.2(0-22)$ in the first trimester, $1.4 \pm 2.7(0-16)$ in the second, $1.5 \pm 3.3(0-20)$ in the third and $3.5 \pm 5.4(0-26)$ in the post-partum period. Mean postpartum SLEDAI-2K score was higher compared to the mean pregnancy SLEDAI- $2 \mathrm{~K}$ score $(\mathrm{p}<0.05) .79 \%$ of all pregnancies sustained LLDAS and $\% 19$ percentage of pregnancies resulted in flares of which $42 \%$ were serious and 58 mild-moderate in severity. $49 \%$ of severe flares occurred during postpartum period, significantly higher compared to all trimesters $(p<0.05)$. Most of the flares had mucocutaneous(\%37), renal(35\%) and haematologic $(25 \%)$ involvement.

APO emerged in $34 \%$ of pregnancies. APO (+) group had significantly longer disease duration compared to APO (-) group $(142 \pm 70$ vs $170 \pm 88 \mathrm{mn}, \mathrm{p}<0.05)$ and higher disease activity during all periods. $\%$ of patients with severe disease activity was significantly low in APO (-) GROUP (\% 18 vs $35, \mathrm{p}<0.05)$ and $\%$ with LLDAS was much higher $(\%$ 88 vs 70$)$.

Conclusion Postpartum period has the highest risk for disease during SLE pregnancies. Active disease during pregnancy increases the risk of APO. Patients with sustained LLDAS have significantly lower APO rates. For a positive pregnancy outcome control of disease activity both during pregnancy and postpartum is required. 